\title{
Advertising Design in Food Marketing: Comparing the Effectiveness of Sensory, Functional and Symbolic Ad Content for Product Evaluation: An Abstract
}

\author{
Klaus-Peter Wiedmann, Janina Haase, Jannick Bettels, \\ and Franziska Labenz
}

\begin{abstract}
Advertising represents one of the most important means in marketing. However, there is often uncertainty about how to exploit advertising most effectively, for example, which content to set to convince the consumer best. This paper examines the influence of ad content on product evaluation in the context of food products. For ad content, we consider two ad elements, text (either comprising sensory, functional or symbolic messages) and picture. To check for significant differences, two studies are performed. Study 1 only incorporates text; Study 2 investigates the three combinations of text and picture. When only considering advertising text, the results reveal no significant differences, which show the relevance of all three dimensions - sensory, functional and symbolic — for food products. When a product picture is added, the findings do identify significant differences. The data analysis indicates that ad effectiveness increases with the complementarity of text and picture. Accordingly, the combination of the primarily sensory picture and the symbolic text providing the most diverse information scores best. The findings provide new insights on advertising design that food firms can use to enhance the consumer's product evaluation in terms of expected taste, perceived experience and quality, overall attitude and purchase intention.
\end{abstract}

K.-P. Wiedmann $\cdot$ J. Haase $(\bowtie) \cdot$ J. Bettels $\cdot$ F. Labenz

Leibniz University of Hannover, Hannover, Germany

e-mail:wiedmann@m2.uni-hannover.de; haase@m2.uni-hannover.de;

bettels@m2.uni-hannover.de; labenz@m2.uni-hannover.de 